\title{
Artesunate v. quinine for severe malaria
}

To the Editor: Severe malaria is a serious and deadly complication of Plasmodium falciparum malaria. ${ }^{[1]}$ We searched the literature to identify the current most efficacious pharmacotherapy for severe malaria. A recent Cochrane meta-analysis ${ }^{[2]}$ compared the efficacy of quinine with artesunate for severe malaria. The primary outcome was all-cause death. The meta-analysis included 8 trials. Artesunate reduced the risk of death both in adults (relative risk (RR) 0.61, 95\% confidence interval (CI) 0.50 - 0.75; 1664 participants, 5 trials) and in children (RR 0.76, 95\% CI 0.65 - 0.90; 5765 participants, 4 trials). In addition, artesunate was less likely to cause hypoglycaemia. ${ }^{[2]}$

Currently, artesunate is not registered for use in South Africa, despite requests to the manufacturer from South African authorities since 2007. However, it is available via the Medicines Control Council of South Africa on a named patient basis. The Parenteral Artesunate Access Programme was launched in South Africa in 2010. ${ }^{[3]}$ The access programme is a valiant effort to improve accessibility, but the programme remains unfunded. The current financial limitations of the access programme prohibit widespread availability of artesunate. The SA Malaria Elimination Committee is addressing these difficulties with the various stakeholders.

Given the high mortality of severe malaria and the superior safety, efficacy, and ease of administration of artesunate, we keenly await its availability at all South African hospitals that are likely to admit patients with malaria.

We thank Professor K Barnes of the Division of Clinical Pharmacology, Department of Medicine, University of Cape Town, for providing information on the current state of affairs with regard to artesunate availability in South Africa.

\section{P Naidoo}

S Saman

Department of Internal Medicine, University of KwaZulu-Natal, and Port Shepstone Regional Hospital, KwaZulu-Natal, South Africa

poobalan1naidoo@yahoo.com

1. Trampuz A, Jereb M, Muzlovic I, Prabhu RM. Clinical review: Severe malaria. Crit Care 2003;7(4):315323.

2. Sinclair D, Donegan S, Isba R, Lalloo DG. Artesunate versus quinine for treating severe malaria. Cochrane Database Syst Rev. 2012 Jun Cochrane Database Syst Rev. 2012 Jun 13; 6:CD005967. [http:// dx.doi.org/10.1002/14651858.CD005967.pub4]

3. Visser Kift E, Kredo T, Barnes KI. Parenteral artesunate access programme aims at reducing malaria fatality rates in South Africa. S Afr Med J 2011;101(4):240-241. 